Check for updates

Cite this: J. Mater. Chem. C, 2021 9, 8640

Received 22nd April 2021 Accepted 26th May 2021

DOI: $10.1039 / \mathrm{d} 1 \mathrm{tc0} 01868 \mathrm{j}$

rsc.li/materials-c

\section{Interplay between ferroelectricity and metallicity in $\mathrm{BaTiO}_{3}^{\dagger}$}

\author{
Veronica F. Michel, (D) Tobias Esswein (D) * and Nicola A. Spaldin (D)
}

\section{Introduction}

Ferroelectricity and metallicity are generally considered to be contra-indicated properties since the metallic charge carriers screen the long-range interactions that favor the ferroelectric structural distortion. ${ }^{1}$ These two properties can nevertheless be combined in systems where the interaction between the itinerant electrons and the polar distortion is weak, as shown theoretically by Anderson and Blount in 1965. Such materials were called "ferroelectric metals", although the term is somewhat ambiguous: Ferroelectricity is in fact defined for materials that show a spontaneous polarization that is switchable by an applied electric field; in the presence of metallic charge carriers however, the electric field is screened by the itinerant electrons and induces an electric current rather than a polarization switch, ${ }^{3,4}$ so that a ferroelectric can formally not be metallic. A more rigorous term is perhaps polar metal, which is a material, such as $\operatorname{LiOsO}_{3},{ }^{5}$ that has a polar crystal class combined with a non-zero density of states at the Fermi level. ${ }^{6}$ In addition to their fundamental interest, polar metals are promising for a range of applications. Some of them are reported to show giant optical responses, and could thus be used in optoelectronic devices. ${ }^{7}$ They are also good candidates for the design of materials with tunable metalinsulator transitions. ${ }^{8}$ Furthermore, they are of relevance for polar

Materials Theory, Department of Materials, ETH Zürich, Wolfgang-Pauli-Strasse 27, 8093 Zürich, Switzerland. E-mail: tobias.esswein@mat.ethz.ch

$\dagger$ Electronic supplementary information (ESI) available: Details on structures, polarizations, and densities of states. See DOI: $10.1039 / \mathrm{d} 1 \mathrm{tc} 01868 \mathrm{j}$ superconductors, which have the potential to show unconventional superconducting states. ${ }^{9}$

Barium titanate $\left(\mathrm{BaTiO}_{3}\right)$ is the prototypical ferroelectric material. It has the ideal $\mathrm{ABO}_{3}$ perovskite structure at high temperatures, corresponding to a centrosymmetric cube with formally Ba cations at the corners, $\mathrm{Ti}^{4+}$ at the center and $\mathrm{O}^{2-}$ at the face centers. ${ }^{10}$ Upon lowering the temperature, three phase transitions occur: at $393 \mathrm{~K}$ a phase transition from a paraelectric cubic to a ferroelectric tetragonal phase is observed, followed by a transition to a ferroelectric orthorhombic phase at $278 \mathrm{~K}$ and to a ferroelectric rhombohedral phase, with polarization along a $\langle 111\rangle$ direction, at $183 \mathrm{~K} .^{10}$ Multiple efforts have been made to make $\mathrm{BaTiO}_{3}$ metallic. In 2008, an insulator-metal transition was reported in $\mathrm{BaTiO}_{3}$ doped with oxygen vacancies $\left(\mathrm{BaTiO}_{3-\delta}\right){ }^{11}$ which was later shown to retain its ferroelectric structural distortion up to an electron concentration $n \approx 1.9 \times 10^{21} \mathrm{~cm}^{-3} \cdot{ }^{12}$ This work was challenged by Jeong et al., who reported, based on neutron diffraction studies, that the ferroelectric ordering and the metallic conduction are not coexisting but rather form two distinct phases in $\mathrm{BaTiO}_{3-\delta} \cdot{ }^{13}$ Recently, Cordero et al. showed through elastic response studies that the ferroelectric transitions persist in metallic $\mathrm{BaTiO}_{3-\delta},{ }^{3}$ confirming the observations of ref. 12. In addition to the introduction of oxygen vacancies, doping in $\mathrm{BaTiO}_{3}$ can be achieved through atomic substitutions, the most common being $\mathrm{La}^{3+}$ for $\mathrm{Ba}^{2+}$ and $\mathrm{Nb}^{5+}$ for $\mathrm{Ti}^{4+}$. Both La- and Nb-doped $\mathrm{BaTiO}_{3}$ show electrical conductivity with a polaronic conduction mechanism, ${ }^{14,15}$ with the polaron formation in Nb-doped $\mathrm{BaTiO}_{3}$ believed to come from 
incoherent B-site off-centering resulting from the random $\mathrm{Nb}$ substitution. ${ }^{16}$

A few computational investigations on doped $\mathrm{BaTiO}_{3}$ have been reported in the last decade. Wang et al. and Iwazaki et al. studied metallic $\mathrm{BaTiO}_{3}$ with the background-charge method in 2012 and showed that the ferroelectric displacements are sustained up to 0.11 electrons per unit cell ( 0.11 e per u.c.). ${ }^{4,17} \mathrm{~A}$ drawback of those works is that the calculations were performed with fully relaxed lattice constants, that were shown to be ill-defined with the background-charge method by Bruneval et al. in 2015. ${ }^{18}$ The specific effects of doping on chemical bonding and the effect of electron-doping on ferroelectricity and phonon dynamics of $\mathrm{BaTiO}_{3}$ were discussed by Hickox-Young et al. and Gu et al., respectively. ${ }^{19,20}$

In 2016, Benedek et al. compared the effect of doping in $\mathrm{LiOsO}_{3}$ and $\mathrm{ATiO}_{3}$ perovskites $(\mathrm{A}=\mathrm{Ba}, \mathrm{Sr}, \mathrm{Ca}){ }^{6}$ They showed that the ferroelectricity is suppressed by electron doping in $\mathrm{BaTiO}_{3}$, whereas the non-centrosymmetricity in metallic $\mathrm{LiOsO}_{3}$ and pseudocubic $\mathrm{CaTiO}_{3}$ (with octahedral rotations not allowed) is robust to addition of charge carriers because of the local-bonding nature of the mechanism underlying the off-centering of the ions. Recently, a meta-screening effect was proposed by Zhao et al. as the main factor determining the persistence of the polar phase in metallic ferroelectrics. ${ }^{21}$

In this work, we explore theoretically the interplay between the contra-indicated properties of ferroelectricity and metallicity in $\mathrm{BaTiO}_{3}$. Using first-principles density functional theory, we address the question of how the ferroelectric B-site off-centering and the likelihood of polarization switchability are affected by metallic charge carriers in $\mathrm{BaTiO}_{3}$. We investigate both electron and hole doping in $\mathrm{BaTiO}_{3}$ first through the background-charge method and subsequently by introducing explicit dopants ( $\mathrm{La}, \mathrm{Nb}, \mathrm{V}, \mathrm{K}, \mathrm{Al}$ and $\mathrm{Sc}$ ). The dopants are chosen based on experimental feasibility and allow us to separate the effects of dopant size, substitution site as well as second-order Jahn-Teller (SOJT) activity on the polarization.

\section{Computational method}

Our calculations are performed using density functional theory (DFT) as implemented in the VASP code ${ }^{22}$ with recommended projector-augmented wave potentials and the PBEsol exchangecorrelation functional. ${ }^{23} \mathrm{We}$ include plane waves up to a kinetic energy cutoff of $600 \mathrm{eV}$. All the used $k$-point grids are gamma-centered; details on the sampling meshes are given below. We converge total free energies to $10^{-6} \mathrm{eV}$ and relax atomic positions until all force components converge below $10^{-3} \mathrm{eV} \AA^{-1}$.

Crystal structure relaxations are computed for both single unit cells and supercells of $\mathrm{BaTiO}_{3}$. We consider both tetragonal and cubic $\mathrm{BaTiO}_{3}$ single unit cells, corresponding to the room-temperature and high-temperature structures, respectively. We compute the tetragonal $\mathrm{BaTiO}_{3}$ single unit cell structural relaxation as follows: We start with the experimental hightemperature, cubic structure and fully relax its atomic positions and lattice constants with a $6 \times 6 \times 6 k$-points grid, while retaining the cubic symmetry. We subsequently displace the $\mathrm{Ti}$ atom by $1 \%$ along the $c$ direction and fully relax the atomic positions and the lattice constants with a $12 \times 12 \times 12 \mathrm{k}$-point grid, while retaining the tetragonal symmetry. We obtain $a=b=$ $3.967 \AA$ and $c=4.065 \AA$; these lattice constants are consistent with experimental literature values for the room-temperature tetragonal phase. ${ }^{24}$ We apply the same procedure for the cubic $\mathrm{BaTiO}_{3}$ single unit cell but fix the lattice constants for the second structural relaxation. This keeps the cubic equal lattice constants while allowing for a lower internal symmetry to permit a polar distortion; we refer to this unit cell as pseudocubic throughout the manuscript. We construct the supercells as multiples of the single unit cell in the $a, b$ and $c$ directions and we investigate $2 \times 2 \times 2$ supercells in detail, for which we use a $12 \times 12 \times 12 k$-point grid.

We compute densities of states for $\mathrm{BaTiO}_{3}$ single unit cells with a $24 \times 24 \times 24 k$-point grid. Crystal Orbital Hamiltonian populations are calculated using the LOBSTER package. ${ }^{25-30}$ Our basis sets for COHP calculations are Ba $(5 \mathrm{~s}, 5 \mathrm{p}, 5 \mathrm{~d}, 6 \mathrm{~s}), \mathrm{Ti}$ $(3 \mathrm{~s}, 3 \mathrm{p}, 3 \mathrm{~d}, 4 \mathrm{~s})$ and $\mathrm{O}(2 \mathrm{~s}, 2 \mathrm{p}) . \mathrm{A} 16 \times 16 \times 16 k$-point grid is used for those calculations.

We dope the $\mathrm{BaTiO}_{3}$ single unit cells with the backgroundcharge method, in which the number of electrons in the system is manually adapted to the desired doping level and a uniform background charge is added to enforce charge neutrality. ${ }^{31}$ Note that the relaxation of lattice constants is not welldefined when using the background-charge method, ${ }^{18}$ so we keep them fixed. We dope supercells by introducing impurity atoms and allowing for relaxation of the lattice constants.

The presented "polarization" values are calculated using

$$
\delta P=\frac{e}{\Omega} \sum_{i} Z_{i}^{*} d_{i}
$$

where $e$ is the electronic charge, $\Omega$ the unit cell volume, $Z_{i}^{*}$ the Born effective charge (BEC) of atom $i$, and $d_{i}$ its relative displacement in the polar direction. ${ }^{32}$ The BECs we use are +2.7 for barium, +7.25 for titanium, -5.71 for oxygens that displace parallel to their Ti-O bond direction and -2.15 for oxygens that displace perpendicular to this direction. ${ }^{33}$ This procedure yields a polarization value of $38 \mu \mathrm{C} \mathrm{cm}{ }^{-2}$ for undoped $\mathrm{BaTiO}_{3}$, which is close to the value of $34 \mu \mathrm{C} \mathrm{cm}{ }^{-2}$ that we compute using the Berry-phase method with a $24 \times$ $24 \times 24 k$-point grid (see Fig. S1 in the ESI $\dagger$ ), in agreement with literature values. ${ }^{34,35}$ For impurity-doped supercells we set the BEC of the impurity to that of the atom it substitutes. Note that the polarization of the doped (metallic) systems is ill-defined as it cannot be switched. This quantity does not correspond to a true polarization obtained through a Berry-phase approach, ${ }^{36,37}$ is not necessarily switchable and does not require insulating behavior. Its value reflects, rather, the amount of ferroelectriclike structural distortion present in the system. Throughout this paper, we use the term polarization to refer to this effective polarization, and the term ferroelectricity to refer to the non-centrosymmetric structural distortion, without implying polarization switchability. 


\section{Results and discussion}

3.1 Introduction of background charge into $\mathrm{a} \mathrm{BaTiO}_{3}$ unit cell

In the first part of our study, we investigate the introduction of doping in $\mathrm{BaTiO}_{3}$ through the background-charge method. This approach allows us to isolate the effect of the electronic charge from other influences such as ion size or change in chemistry associated with the introduction of explicit dopant atoms. Consistent with previous literature results, ${ }^{19}$ we find the polarization to be reduced by the introduction of charge carriers, and to be more sensitive to the introduction of electrons than holes, due to the different character of the valence and conduction bands.

3.1.1 Electron doping. The calculated polarization of $\mathrm{BaTiO}_{3}$ as a function of charge-carrier concentration is shown in the top panel of Fig. 1, for both electron and hole doping, and tetragonal and cubic unit cells. We discuss the electrondoped tetragonal system first. On electron addition, the polarization of tetragonal $\mathrm{BaTiO}_{3}$ decreases until it is completely suppressed at 0.2 e per u.c.

The decrease in polarization can be understood through simple electronic structure considerations: in Fig. 2 we show the calculated density of states in the region of the Fermi energy for tetragonal $\mathrm{BaTiO}_{3}$ doped with 0.5 e per u.c. We see that the valence band has mainly $\mathrm{O} 2 \mathrm{p}$ character, whereas the conduction band has mainly Ti $3 \mathrm{~d}$ character and the barium ions have no significant contribution to the DOS in the region of the band gap. Compared to pure $\mathrm{BaTiO}_{3}$ (not shown), the DOS is not strongly affected by the charge carriers, apart from the shift of the Fermi level into the conduction band.

The added electrons occupying primarily the Ti $3 \mathrm{~d}$ energy levels can also be seen clearly in the charge densities of Fig. 3, where the gray surfaces indicate the added electron density. With increasing electron doping, we see that the charge density on the $\mathrm{Ti} 3 \mathrm{~d}$ orbitals progressively increases and as a result the $\mathrm{Ti}$ ion deviates further from its formally $\mathrm{d}^{0}$ electron configuration. Correspondingly, the off-centering described by the SOJT effect, which is favored for $\mathrm{d}^{0}$ electronic configurations, is reduced. ${ }^{38}$

We compute integrated Crystal Orbital Hamiltonian Populations (ICOHPs) to confirm our explanation for the polarization decrease with increasing electron concentration. The bottom of Fig. 1 shows the ICOHP as a function of the charge-carrier concentration for the Ti-O axial top, axial bottom and equatorial bonds (these are also illustrated in Fig. 1). All the values are normalized to their respective value in the centrosymmetric structure. In the polar structure, the Ti is off-centered along $c$, resulting in a strong, short Ti-O axial top bond with a high ICOHP value and a weak, long bottom bond with a low ICOHP value. Upon adding electrons, the top bond is weakened and its ICOHP value is lowered. Conversely, the bottom bond is strengthened and its ICOHP value increases. The Ti-O equatorial bonds are not particularly affected by the structural distortion decrease.

3.1.2 Hole doping. In contrast to electron doping, the introduction of holes in tetragonal $\mathrm{BaTiO}_{3}$ causes first an increase in polarization (from 0 to $0.2 \mathrm{~h}$ per u.c., as seen in
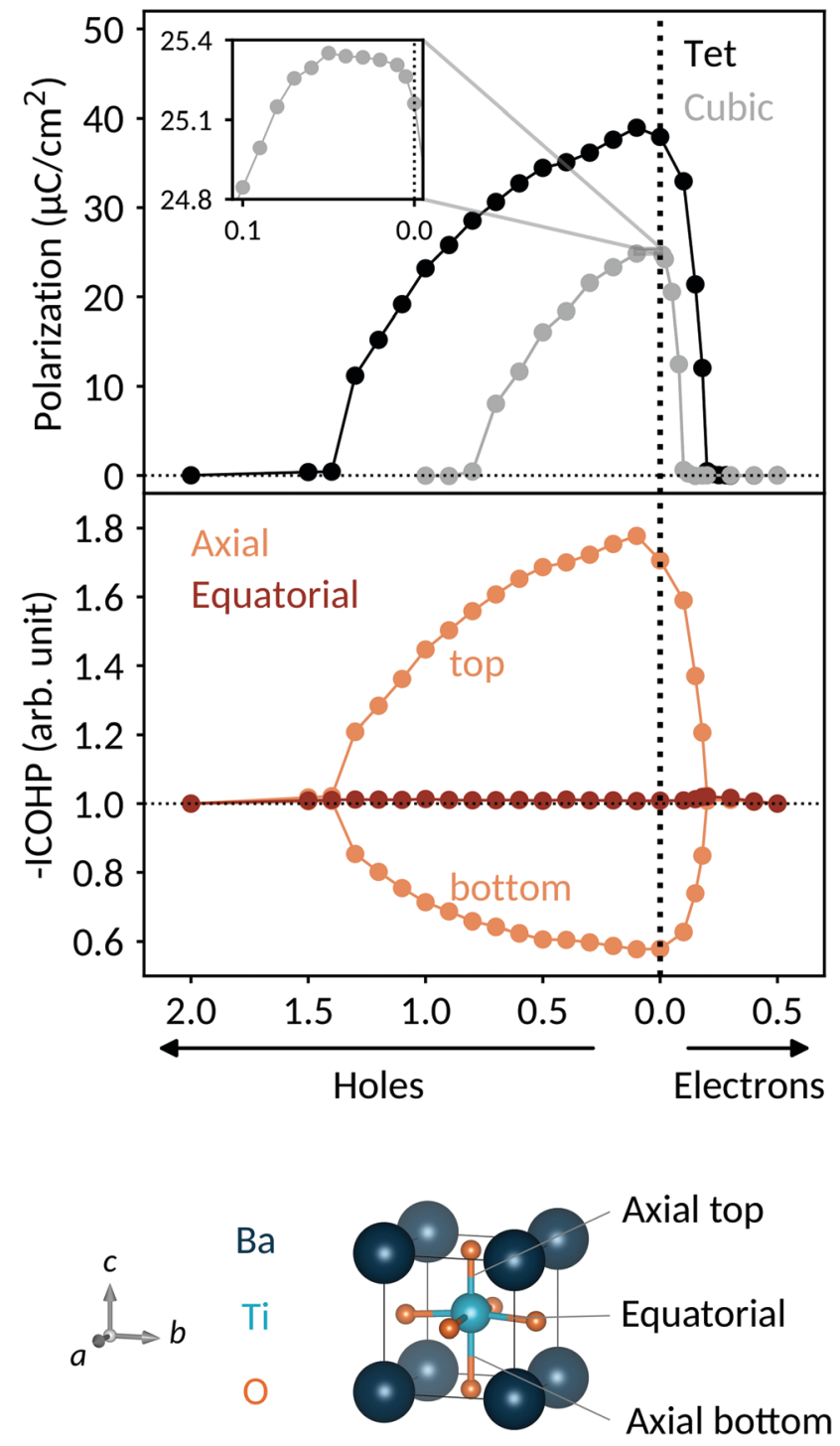

Fig. 1 Top: Polarization as a function of the charge-carrier concentration (electrons on the right, holes on the left) for tetragonal and pseudocubic (abbreviated tet and cubic) $\mathrm{BaTiO}_{3}$. The pseudocubic symmetry refers to equal lattice constants with an allowed lower internal symmetry so that a polar distortion can occur. We see that the polarization is reduced on electron doping for all concentrations. The addition of holes first increases the polarization before reducing it, with a slower polarization suppression than in the electron doping case. The pseudocubic and tetragonal systems behave in a very similar manner, although the pseudocubic system has lower polarization values for the same charge-carrier concentration. Bottom: Integrated Crystal Orbital Hamiltonian Populations (ICOHPs) for the $\mathrm{Ti}-\mathrm{O}$ bonds of tetragonal $\mathrm{BaTiO}_{3}$ as a function of the charge-carrier concentration. Three Ti-O bonds are considered and showed in the figure: the Ti-O axial top, axial bottom and equatorial. All ICOHPs are normalized to their value in the centrosymmetric structure. The higher the ICOHP value, the stronger its corresponding bond.

the top of Fig. 1), followed by a decrease ( $>0.2 \mathrm{~h}$ per u.c.) and complete suppression at $1.5 \mathrm{~h}$ per u.c. Overall, the amount of charge carriers needed to suppress the polar distortion is a factor 7.5 larger for hole doping than for electron doping.

The particular behavior of the hole-doped systems can be understood by considering their detailed densities of states. 


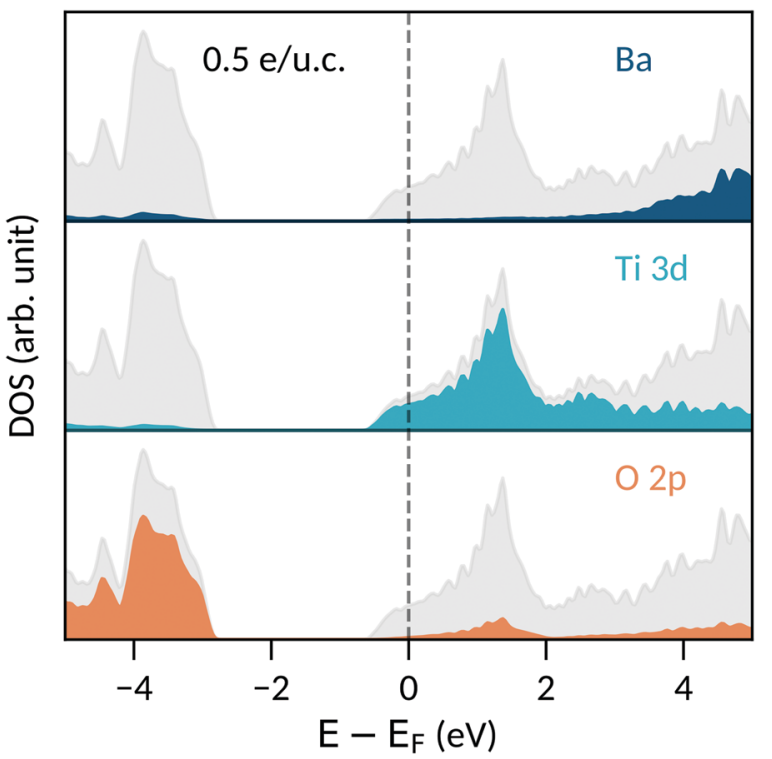

Fig. 2 Density of states for tetragonal $\mathrm{BaTiO}_{3}$ doped with 0.5 e per u.c. The total DOS is shown in gray and the $\mathrm{Ba}, \mathrm{Ti} 3 \mathrm{~d}$ and $\mathrm{O} 2 \mathrm{p}$ contributions are shown in blue, teal and orange, respectively. The valence band has mainly $\mathrm{O} 2 \mathrm{p}$ character, whereas the conduction band has a high $\mathrm{Ti} 3 \mathrm{~d}$ contribution. The Fermi energy, $E_{\mathrm{F}}$, lies close to the bottom of the conduction band due to the addition of electrons.

Fig. 4 shows the oxygen site-resolved DOS for 0.1 and $0.5 \mathrm{~h}$ per u.c., with axial and equatorial sites shown separately. On removing electrons, the Fermi energy shifts down into the valence band, corresponding to a charge depletion of the oxygen atoms. For doping concentrations less than $0.1 \mathrm{~h}$ per u.c., we see (Fig. 4, top panel) that electrons are largely only removed from the equatorial oxygen band, and the axial oxygens are almost unaffected. We will come back to this point below. For higher charge-carrier concentrations, depletion from the axial oxygen atoms starts to take place. We show in Fig. 4 the case of $0.5 \mathrm{~h}$ per u.c. and see that the Fermi energy lies in both the axial and equatorial bands. Again invoking the SOJT effect, in which axial oxygens transfer electrons to the empty Ti d orbitals and stabilize the off-centering, we see that removing electrons from the axial oxygens reduces the tendency for the polar structural distortion to occur. The evolution of the electron depletion from equatorial to axial oxygens with increasing hole concentration can also be seen in the charge densities of Fig. 3.

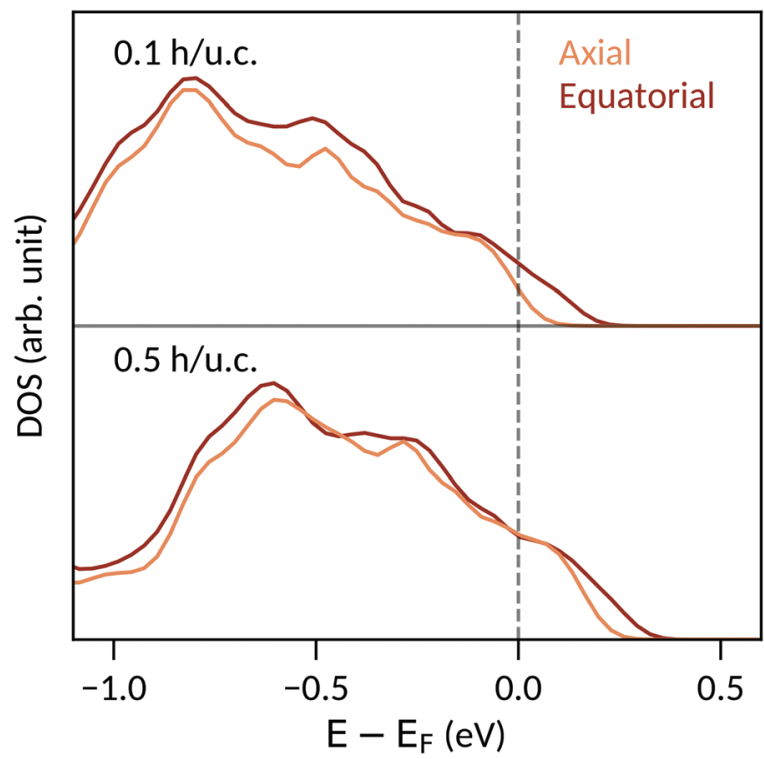

Fig. 4 Oxygen site-resolved densities of states for tetragonal $\mathrm{BaTiO}_{3}$ doped with $0.1 \mathrm{~h}$ per u.c. (top) and $0.5 \mathrm{~h}$ per u.c. (bottom). At $0.1 \mathrm{~h}$ per u.c., electrons are primarily removed from the equatorial oxygen atoms. At $0.5 \mathrm{~h}$ per u.c., the axial oxygen atoms are also strongly depleted.

Next we consider the ICOHP which is plotted as a function of the hole concentration in Fig. 1. This allows us to understand the polarization increase at small hole concentrations, where charge depletion from the equatorial oxygen is dominant. This electron depletion weakens the equatorial Ti-O bonds, reduces the tension in the equatorial plane, and allows the polar displacements along $c$ to increase. (Note that the equatorial bond weakening is not resolved in the equatorial Ti-O ICOHPs because of its small amount). Correspondingly, between 0 and $0.2 \mathrm{~h}$ per u.c., the $\mathrm{Ti}-\mathrm{O}$ axial bottom bond is weakened and the Ti-O axial top bond is strengthened, as seen in their respective decreasing and increasing ICOHP values. For $0.3 \mathrm{~h}$ per u.c. and larger, a behavior similar to the electron doping case is observed, consistent with the observed loss of polarization. The ICOHP values for the Ti-O axial top bond follow the trend of the polarization values, indicating a direct relation between changes in polarization and Ti-O bond strength.

3.1.3 Influence of unit cell shape. Next we investigate the influence of the unit cell shape by comparing the behavior of
$0.3 \mathrm{~h} / \mathrm{u} . \mathrm{c}$

$0.2 \mathrm{~h} /$ u.c.

0.1 h/u.c.
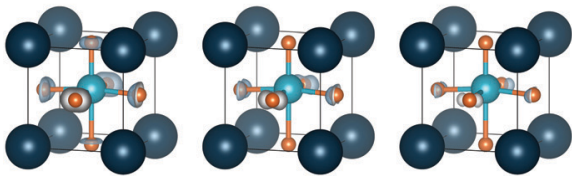

Increasing hole concentration
0.1 e/u.c.

$0.2 \mathrm{e} /$ u.c.

0.5 e/u.c.

$\mathrm{Ba}$

$\mathrm{Ti}$

$\mathrm{O}$

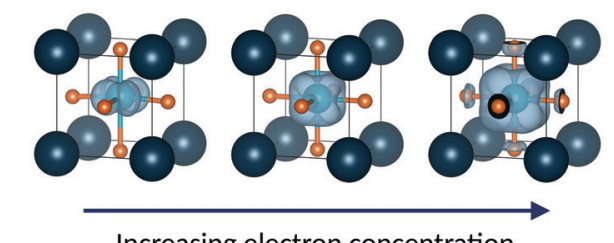

Increasing electron concentration

Fig. 3 Conduction-band electron charge density (right) and valence-band hole charge density (left) for tetragonal $\mathrm{BaTiO}_{3}$. For electron doping, the gray surfaces all have the same isosurface level (charge $\approx 7.5 \times 10^{-4}$ e) and show the location of the added electrons. For hole doping, the gray surfaces also have the same isosurface level, which is one order of magnitude larger than for electrons (charge $\approx 7.3 \times 10^{-3}$ e), and reveal where the electron depletion occurs. 
tetragonal and pseudocubic (abbreviated tet and cubic in Fig. 1) $\mathrm{BaTiO}_{3}$. Note that, as mentioned above, the pseudocubic system has equal lattice parameters and therefore cubic shape, but we allow for a lower internal symmetry to permit a polar distortion. The tetragonal and pseudocubic systems follow the same trends, as seen in the top of Fig. 1. Their similarity can be explained by their comparable electronic structures (the DOS for the pseudocubic system can be found in Fig. S2 and S3 of the ESI $\dagger$ ). The polarization of the pseudocubic system is, however, smaller than the tetragonal one and its polarization is suppressed faster. This is related to the absence of coupling to strain, and correspondingly smaller space in the $c$ direction, resulting in smaller atomic displacements and thus a smaller polarization. In the rest of the paper, we present only the results for tetragonal $\mathrm{BaTiO}_{3}$. Note that we focus on the tetragonal symmetry to provide guidelines on the behaviour of $\mathrm{BaTiO}_{3}$ at room temperature, and that we do not expect the mechanisms to be particularly different for other symmetries, for example the rhombohedral that occurs at low temperatures.

\subsection{Introduction of impurity atoms in $\mathrm{BaTiO}_{3}$ supercells}

In the second part of our study, we include explicit impurity atoms in tetragonal $\mathrm{BaTiO}_{3}$ and investigate their effect on the polarization of the system. We consider electron and hole doping through A-site as well as B-site substitution. For electron doping, we introduce La on the A site and $\mathrm{Nb}$ or $\mathrm{V}$ on the $\mathrm{B}$ site. We explore hole doping through the introduction of $\mathrm{K}$ on the $\mathrm{A}$ site and $\mathrm{Al}$ or Sc on the $\mathrm{B}$ site. All dopants of interest add or remove one charge carrier per $2 \times 2 \times 2$ supercell, resulting in carrier concentrations of 0.125 carriers per u.c. Results for smaller charge-carrier concentrations can be found in Fig. S4 of the ESI. $\dagger$ Note that we consider only single substitutional impurities that introduce either one electron or one hole in their usual formal charge state. We do not investigate vacancies, the introduction of multiple defects or impurity-vacancy complexes.

For every $\mathrm{X}-\mathrm{BaTiO}_{3}$ system (where $\mathrm{X}$ denotes a general dopant), we perform three different calculations in order to identify and separate contributions to the polarization change. The first calculation consists of a structural relaxation of the internal coordinates of $\mathrm{X}-\mathrm{BaTiO}_{3}$ containing the impurity atom but not its corresponding charge carriers and with lattice constants fixed to their undoped values. We achieve this by compensating the charge carriers added with the dopant by background-charge doping (the background charge requires the use of fixed lattice constants). We refer to this component as the impurity-atom contribution. In the second calculation we remove the compensating background-charge and relax the internal coordinates of $\mathrm{X}-\mathrm{BaTiO}_{3}$ with the impurity atom and its charge carriers, still keeping the lattice constants fixed. We refer to this as the charge-carrier contribution. In the third calculation, we make a full structural relaxation of both the internal coordinates and lattice parameters of $\mathrm{X}-\mathrm{BaTiO}_{3}$, containing the impurity atom with its charge carriers; we call this situation free lattice constants and we refer to this third component as the lattice-constant contribution. This last system corresponds to the most realistic one. These three scenarios allow us to separate the contributions to the polarization change coming from the impurity atom, from the charge carriers and from changes in lattice constants. In the next sections, we present a detailed analysis of these contributions for the different impurity-doped $\mathrm{BaTiO}_{3}$ systems. A compact overview of the discussed systems and features can be found in Table 1. The polarization changes arising from the different contributions are illustrated in Fig. 5. Background-charge results are given as a reference in both cases and all given polarization changes $\Delta P$ in $\%$ refer to the changes compared to the original $\mathrm{BaTiO}_{3}$ polarization.

Note that all the listed Shannon ionic radii are taken from ref. 39 and 40 and that tolerance factors are calculated with these values, with the charge of the impurity ion taken as its formal charge when introduced as a dopant - e.g. $5+$ for $\mathrm{Nb}-$ with the coordination number appropriate to the substituted site (12 for the A site and 6 for the B site).

\subsubsection{Donor doping}

\subsubsection{A-Site dopants}

We start by considering the La-doped $\mathrm{BaTiO}_{3}$ system $\left(\mathrm{Ba}_{1-x} \mathrm{La}_{x}\right.$ $\mathrm{TiO}_{3}$, BLTO). The La substitutes on the A site as formally trivalent $\mathrm{La}^{3+}$, which has a smaller ionic radius than $\mathrm{Ba}^{2+}$ and, as we discuss below, influences therefore the structure of the system.

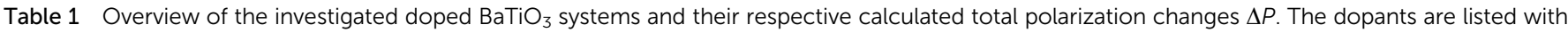

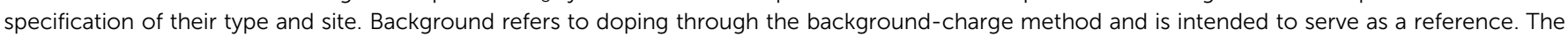

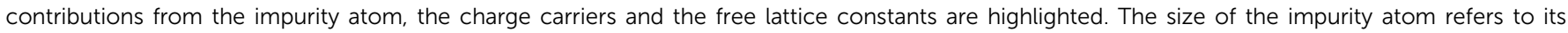

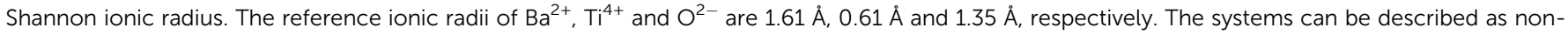
polar or polar

\begin{tabular}{|c|c|c|c|c|c|c|c|c|c|c|}
\hline \multirow[b]{2}{*}{ Doping type } & \multirow[b]{2}{*}{ Dopant } & \multirow[b]{2}{*}{ Site } & \multicolumn{2}{|c|}{ Impurity atom } & \multicolumn{2}{|c|}{ Charge carriers } & \multicolumn{2}{|c|}{ Lattice constants } & \multirow[b]{2}{*}{ Total $\Delta P(\%)$} & \multirow[b]{2}{*}{ Description } \\
\hline & & & Size $(\AA)$ & SOJT & Type & Localization & Volume & Tetragonality & & \\
\hline \multirow[t]{4}{*}{ Donors } & Background & - & - & - & $\mathrm{e}^{-}$ & No & - & - & -29 & - \\
\hline & $\mathrm{La}^{3+}$ & A & 1.36 & - & $\mathrm{e}^{-}$ & No & $\downarrow$ & Loss & -100 & Non-polar \\
\hline & $\mathrm{Nb}^{5+}$ & B & 0.64 & Yes & $\mathrm{e}^{-}$ & Yes & Const. & Loss & -58 & Polar \\
\hline & $\mathrm{V}^{5+}$ & B & 0.54 & Yes & $\mathrm{e}^{-}$ & Yes & $\downarrow$ & Loss & -30 & Polar \\
\hline \multirow[t]{4}{*}{ Acceptors } & Background & - & - & - & $\mathrm{h}^{+}$ & No & - & - & +2 & - \\
\hline & $\mathrm{K}^{+}$ & A & 1.64 & - & $\mathrm{h}^{+}$ & No & Const. & Gain & +24 & Polar \\
\hline & $\mathrm{Al}^{3+}$ & $\mathrm{B}$ & 0.54 & No & $\mathrm{h}^{+}$ & No & $\downarrow$ & Loss & -36 & Polar \\
\hline & $\mathrm{Sc}^{3+}$ & $\mathrm{B}$ & 0.75 & Yes & $\mathrm{h}^{+}$ & No & $\uparrow$ & Loss & -19 & Polar \\
\hline
\end{tabular}




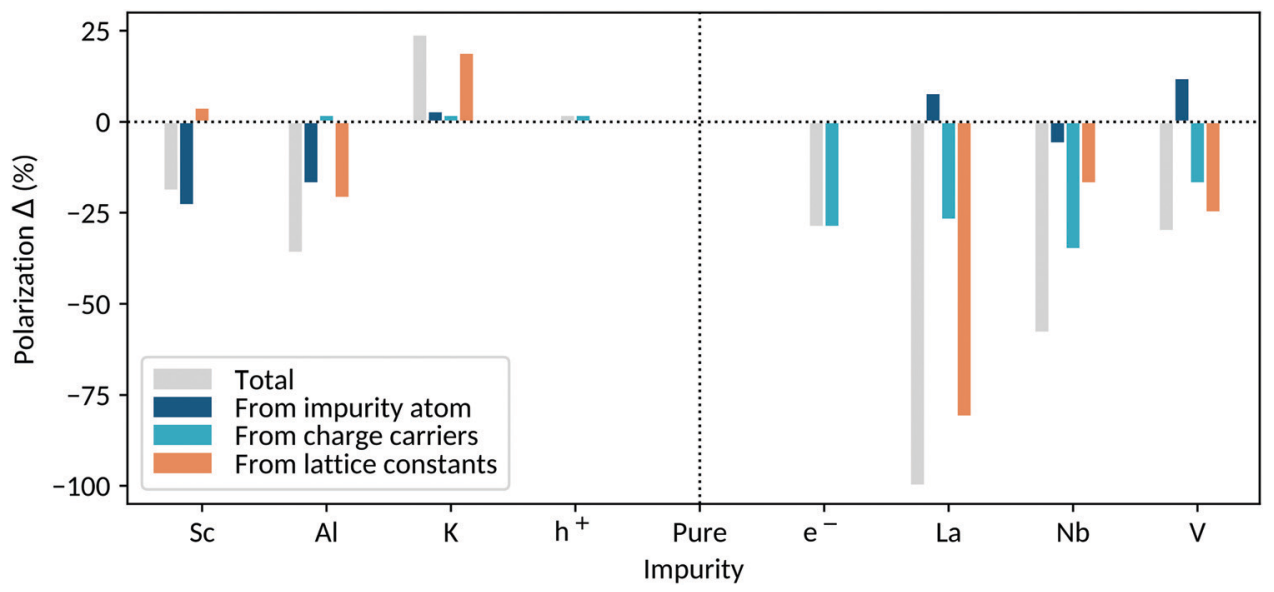

Fig. 5 Polarization difference caused by the introduction of doping in tetragonal $\mathrm{BaTiO}_{3}$. All the systems have a charge-carrier concentration of 0.125 carriers per u.c., corresponding to one impurity atom in a $2 \times 2 \times 2$ supercell. Positive polarization $\Delta$ values ( $y$ axis) correspond to a polarization gain, negative ones to a polarization loss. The total polarization difference (gray) is split into contributions corresponding to: (i) the effect of the impurity atom without its carriers (dark blue). (ii) The effect of the introduced charge carriers (teal). (iii) The effect of the changing shape and volume of the system (orange). The total polarization change is obtained as the sum of the competing contributions present in the system.

Impurity-atom contribution. When a La atom without its respective charge carriers is added to $\mathrm{BaTiO}_{3}$, we find that the polarization of the system increases by $8 \%$, due to a displacement of the small $\mathrm{La}^{3+}$ cation in the $c$ direction. Besides directly contributing to the polarization of the system, the La also has an influence on the Ti sites in two ways: First, the La is underbound and pulls the Ti atoms immediately above it towards itself, reducing their off-centering. Second, the La displacement along $c$ leaves more space for the $\mathrm{Ti}$ below it to off-center; this contribution dominates over the first.

Charge-carrier contribution. Upon adding the electrons, the polarization of the system is reduced by $27 \%$ compared to the original $\mathrm{BaTiO}_{3}$ polarization. This value is close to that found in the background-charge doped reference (29\%). In fact, the introduction of the La does not influence the density of states of the system. The La f states lie high in energy and do not affect the region around the Fermi energy; the bottom of the conduction band therefore has pure $\mathrm{Ti}$ character and the electrons distribute homogeneously over the B sites of the system.

Lattice-constant contribution. Finally we consider the relaxation of the lattice constants. Here we find that the decreases in both volume and tetragonality, due to the small La size, reduce the polarization by a further $81 \%$.

Summary. The introduction of La in $\mathrm{BaTiO}_{3}$ results in a complete polarization suppression with all the atoms adopting their centrosymmetric positions. The volume and tetragonality decrease caused by the small La size are the main factors dominating the polarization loss. Experimental reports claim that the conduction mechanism in BLTO is of polaronic nature. ${ }^{14}$ No polaronic localization is seen in the above presented results. This could be due to an incomplete description of the charge imbalance compensation mechanisms present in the system. We consider only an electronic compensation of
$\mathrm{Ba}^{2+} \Rightarrow \mathrm{La}^{3+}+\mathrm{e}^{-}$, without any further ionic contributions as reported in the literature (e.g. $\left.4 \mathrm{Ba}^{2+}+\mathrm{Ti}^{4+} \Rightarrow 4 \mathrm{La}^{3+}+\mathrm{V}_{\mathrm{Ti}}\right) .^{41}$ Furthermore, hybrid functionals might be necessary to capture polaronic behavior, as they have been shown to properly describe polarons coexisting with delocalized electrons in $\mathrm{TiO}_{2} \cdot{ }^{42}$

\subsubsection{B-Site dopants}

We consider $\mathrm{Nb}$ and $\mathrm{V}$ as B-site substituting donor impurities; the respective systems can be written as $\mathrm{BaTi}_{1-x} \mathrm{Nb}_{x} \mathrm{O}_{3}$ (BTNO) and $\mathrm{BaTi}_{1-x} \mathrm{~V}_{x} \mathrm{O}_{3}$ (BTVO). When introduced in $\mathrm{BaTiO}_{3}, \mathrm{Nb}$ and $\mathrm{V}$ are present as $\mathrm{Nb}^{5+}$ and $\mathrm{V}^{5+}$, respectively, and have therefore formally empty d orbitals, making them SOJT active. $\mathrm{Nb}^{5+}(0.64$ $\AA)$ is larger than $\mathrm{Ti}^{4+}(0.61 \AA)$, whereas $\mathrm{V}^{5+}(0.54 \AA)$ is smaller. The off-centering of the two dopants depends on their size and the tolerance factor of the structure.

We begin with the Nb-doped $\mathrm{BaTiO}_{3}$ system:

Impurity-atom contribution. The introduction of the $\mathrm{Nb}$ without its charge carriers decreases the polarization by $6 \%$, due to the reduced off-centering of the $\mathrm{Nb}$ atom and its axial neighboring Ti along the polar axis. The reduced structural distortion is due to the $\mathrm{Nb}$ size: The tolerance factor of $\mathrm{BaNbO}_{3}$ is 1.03 , compared to 1.07 for $\mathrm{BaTiO}_{3}$. Although it has the empty d orbital configuration needed to be SOJT active, it is too big to actually off-center. The $\mathrm{Ti}$ atoms axial to the $\mathrm{Nb}$ are directly affected by the $\mathrm{Nb}$ behavior: Since the $\mathrm{Nb}$ is less off-centered, they have less space to displace in the $c$ direction and are more centrosymmetric.

Charge-carrier contribution. The addition of the electrons reduces the polarization by a further $35 \%$, which is more than expected from the background-charge reference (29\%). The additional suppression results from a slight accumulation of the electrons on the $\mathrm{Nb}$ atom and its axial neighboring Ti along the polar axis, which have the largest contribution to the bottom of the conduction band (Fig. S5 of the ESI $\dagger$ ). The 
electron accumulation heavily reduces the structural distortion of these two sites, increasing the polarization loss due to the presence of the charge carriers.

Lattice-constant contribution. When the lattice constants are relaxed, the polarization is reduced by a further $17 \%$. This polarization change can be assigned to the reduced tetragonality since the volume of the system stays rather constant. Note that this constant volume is not directly intuitive. In fact, $\mathrm{Nb}^{5+}$ is larger than $\mathrm{Ti}^{4+}$, so that a structural expansion would be expected. It is nevertheless counterbalanced by the loss of polarization, resulting in an unchanged volume.

Summary. Nb-doped $\mathrm{BaTiO}_{3}$ remains polar, although with an overall polarization decrease of $58 \%$. The charge carriers are the most important factor contributing to the polarization loss. We find that they tend to accumulate on the $\mathrm{Nb}$ atom and its nearest axial $\mathrm{Ti}$, consistent with the polaronic conduction mechanism proposed in the literature. ${ }^{15,16}$

Next we evaluate the polarization change in the V-doped system where we find that the small size of $\mathrm{V}$ leads to markedly different behavior.

Impurity-atom contribution. The addition of $\mathrm{V}$ without its respective charge carriers induces a polarization increase of 12\%: The $\mathrm{V}$ is smaller than the $\mathrm{Ti}$ and therefore able to off-center more $\left(\mathrm{BaVO}_{3}\right.$ has a large tolerance factor of 1.11). The Ti axial to the $\mathrm{V}$ are also off-centered more because the $\mathrm{V}$ influences its axial environment and induces a cascade offcentering - the same effect but in the reverse direction as that seen for the BTNO system.

Charge-carrier contribution. The introduction of electrons decreases the polarization by $17 \%$ compared to pure $\mathrm{BaTiO}_{3}$, which is less than expected from the background-charge reference (29\%). The added charge carriers strongly localize on the $\mathrm{V}$ because of its very large contribution to the bottom of the conduction band (Fig. S6 of the ESI $\dagger$ ). We might expect this localization to increase the polarization suppression, as seen in the BTNO system. However, in this case the V is so small that it displaces and contributes to the polarization even if its SOJT hybridization stabilization is quenched by the extra electrons. In addition, the electron localization on the $\mathrm{V}$ reduces their accumulation on the $\mathrm{Ti}$ sites, so that their polar structural distortions are not affected much by the doping.

Lattice-constant contribution. The relaxation of the lattice constants results in a further polarization decrease of $25 \%$. This is explained by the reduced volume and tetragonality, due to the small $\mathrm{V}$ size.

Summary. The introduction of $\mathrm{V}$ in $\mathrm{BaTiO}_{3}$ reduces the polarization by overall $30 \%$. The addition of electrons reduces the expected polarization loss compared with the backgroundcharge reference because of charge-carrier localization on the $\mathrm{V}$ site, an effect which has also been reported in the literature. ${ }^{43} \mathrm{~V}$ doping in $\mathrm{BaTiO}_{3}$ has been observed experimentally to increase the polarization for low $\mathrm{V}$ concentrations $(<0.5$ at $\%)$, followed by a polarization loss at higher concentrations, ${ }^{44}$ consistent with our calculations.

\subsubsection{Acceptor doping}

3.2.2.1 A-Site dopants

Now we move to acceptor doping and begin with the substitution of monovalent $\mathrm{K}$ on the $\mathrm{Ba} \mathrm{A}$ site. In the $\mathrm{K}$-doped $\mathrm{BaTiO}_{3}$ system $\left(\mathrm{Ba}_{1-x} \mathrm{~K}_{x} \mathrm{TiO}_{3}, \mathrm{BKTO}\right)$, the $\mathrm{K}$ is present as $\mathrm{K}^{+}$, which is only slightly larger than $\mathrm{Ba}^{2+}$.

Impurity-atom contribution. Adding a $\mathrm{K}$ atom with no additional charge carriers causes a polarization gain of $3 \%$, caused by complex atom rearrangement, which shows the tendency of the system towards tetragonality, as we will further discuss in the Lattice-constant contribution section. This results in a displacement of the $\mathrm{K}^{+}$ion in the $c$ direction, increased displacements of the Ti on the plane below the $\mathrm{K}$ - they have more space to off-center because of the $\mathrm{K}$ displacement - and decreased offcentering of the $\mathrm{Ti}$ above the $\mathrm{K}$.

Charge-carrier contribution. Electron depletion increases the polarization by a further $2 \%$. This is in agreement with the effect observed in the background-charge doped $\mathrm{BaTiO}_{3}$ (2\%). In fact, the introduction of the $\mathrm{K}$ atom has no particular influence on the valence band of the system, which has still $\mathrm{O}$ $2 \mathrm{p}$ character.

Lattice-constant contribution. The lattice constant relaxation induces a further polarization increase of $19 \%$. When adding a $\mathrm{K}$ atom, the volume of the system stays rather constant, as $\mathrm{K}^{+}$is only slightly bigger than the $\mathrm{Ba}^{2+}$. More interestingly, the tetragonality of the system is considerably increased, explaining the polarization increase. This indicates that structural factors affect the polarization of the system primarily through their influence on the polar $c$ axis: the more space in the $c$ direction (rather than the overall volume), the higher the potential for a polar distortion.

Summary. The introduction of $\mathrm{K}$ in $\mathrm{BaTiO}_{3}$ at a concentration $0.125 \mathrm{~h}$ per u.c. overall increases the polarization of the system by $24 \%$, mainly because of the increased tetragonality. We remind the reader that our polarization values are obtained using the Ba Born effective charge of +2.7 for $\mathrm{K}$ and that they are therefore likely to be smaller in practice.

\subsubsection{B-Site dopants}

Finally we move to the B-site hole dopants and consider two impurities: $\mathrm{Al}^{3+}$ which is quite ionic, and $\mathrm{Sc}^{3+}$ which is SOJT active.

We begin with the Al-doped $\mathrm{BaTiO}_{3}\left(\mathrm{BaTi}_{1-x} \mathrm{Al}_{x} \mathrm{O}_{3}, \mathrm{BTAO}\right)$ in which the trivalent $\mathrm{Al}^{3+}$ is smaller than $\mathrm{Ti}^{4+}$; we expect it therefore to have a strong structural influence.

Impurity-atom contribution. Introducing an $\mathrm{Al}$ atom in $\mathrm{BaTiO}_{3}$ without its corresponding charge carriers reduces the polarization by $17 \%$. The $\mathrm{Al}$ atom has in fact a reduced offcentering, since it has no d orbitals that would favour its hybridization with the axial oxygen atom. It is nevertheless still slightly displaced compared to its centrosymmetric position because of its small size. The tolerance factor of pure $\mathrm{BaAlO}_{3}$, 1.11, indicates that the $\mathrm{Al}$ is small enough to rattle. 
The decreased $\mathrm{Al}$ off-centering also reduces the off-centering of the its axial neighboring Ti along the polar axis.

Charge-carrier contribution. Electron depletion increases the polarization by $2 \%$, consistent with the backgroundcharge doped reference and the minimal influence of the $\mathrm{Al}$ on the electronic structure in the region of the Fermi energy. While the electron depletion on the oxygen atoms around the $\mathrm{Al}$ has a small effect, the depleted equatorial oxygens around the $\mathrm{Ti}$ ions cause an increased off-centering of the $\mathrm{Ti}$ as in the background-charge case. The $\mathrm{Ti}$ axial to the $\mathrm{Al}$ are particularly affected, because of the high contribution of the oxygen atoms around them to the top of the valence band, and contribute most to the polarization gain.

Lattice-constant contribution. Upon relaxing the lattice constants, a polarization loss of $21 \%$ occurs. This is due to a decrease of the volume and tetragonality of the system, reducing the B-site off-centering homogeneously.

Summary. The introduction of $\mathrm{Al}$ into $\mathrm{BaTiO}_{3}$ reduces the polarization, overall by $36 \%$. This is mainly due to the non-offcentering of the $\mathrm{Al}$, which has no d orbitals and is therefore not SOJT active, as well as the loss of space through volume contraction and tetragonality decrease.

The last system that we analyze is Sc-doped tetragonal $\mathrm{BaTiO}_{3}\left(\mathrm{BaTi}_{1-x} \mathrm{Sc}_{x} \mathrm{O}_{3}, \mathrm{BTSO}\right)$, where $\mathrm{Sc}$ is present as $\mathrm{Sc}^{3+} \cdot \mathrm{Sc}^{3+}$ is larger than $\mathrm{Ti}^{4+}$ so that we expect it to influence the structure of the system.

Impurity-atom contribution. Adding a Sc without its charge carriers reduces the polarization by $23 \%$. The transition metal sites causing the polarization loss are the Sc site and its axial neighboring Ti along the polar axis. The origin, as in the BTNO system, is the large $\mathrm{Sc}^{3+}$ size: The $\mathrm{BaScO}_{3}$ tolerance factor is 0.99 , meaning that, even though it is SOJT active, the Sc is too big to actually displace in the $c$ direction. The Ti axial to the Sc also off-center less. This is due to the Sc influencing its axial environment; as it is almost centrosymmetric, there is less space for the atoms around it to off-center.

Charge-carrier contribution. Electron depletion increases the polarization by less than $1 \%$, a smaller amount compared to the background-charge doped reference (2\%). The oxygen atoms contributing most to the top of the valence band, and therefore most depleted upon doping, are the equatorial and axial oxygens around the Sc. However, because of its large size, the Sc is not affected by this depletion, reducing the net polarization gain.

Lattice-constant contribution. When the lattice constants of the system are relaxed, the polarization increases by $4 \%$. This is related to the volume expansion due to the $\mathrm{Sc}^{3+}$ being bigger than the $\mathrm{Ti}^{4+}$. The tetragonality of the system stays constant and therefore neither counterbalances the volume effect nor increases it.

Summary. The net polarization loss in BTSO amounts to $19 \%$. This is due to the large size of the Sc counteracting the other factors that would increase the polarization of the system.
3.2.3 Overview impurity doping. Our results suggest that the factors contributing to the polarization change can be divided into three effects: (i) the chemistry and size of the impurity atom, (ii) the charge carriers and their degree of localization, and (iii) changes in tetragonality and volume.

The influence of the impurity atom depends on its size and SOJT activity. On the A site, small dopants tend to increase the polarization by cation displacement (e.g. La). Note that the polarization increase due to doping with small A-site cations cannot be achieved if all the A sites are substituted, where cooperative rotations would be preferred over the polar distortion (as seen in e.g. $\mathrm{SrTiO}_{3}$ ). ${ }^{45}$ On the $\mathrm{B}$ site, off-centering is controlled by the presence of empty d orbitals (for the atom to be SOJT active) and the space available for off-centering, which correlates with the size of the atom. The off-centering of SOJT active atoms can be suppressed if they are too large $(e . g$. Nb and Sc) and does not occur if they lack empty d orbitals in an appropriate energy range (e.g. Al). If both SOJT activity and small size are present, increased off-centering is observed (e.g. V). A theoretically promising (although experimentally inaccessible) avenue would correspond to doping $\mathrm{BaTiO}_{3}$ with a SOJT active acceptor atom that is smaller than Sc. This is however not feasible as $\mathrm{Sc}^{3+}$ is the smallest trivalent SOJT active ion that exists.

The effect of the charge carriers depends on their type and degree of localization. Electrons have a stronger influence on the polarization than holes, as already discussed for the background-charge doped systems. Overall, electrons reduce the polarization, while charge carrier localization can increase (e.g. $\mathrm{Nb})$ or decrease (e.g. $\mathrm{V})$ the extent of the reduction. In contrast, the introduction of holes very slightly increases the polarization of the system at 0.125 carriers per u.c.

The relaxation of the lattice constants permits changes in the tetragonality and volume of the system depending on the introduced dopant. Small atoms tend to induce a volume contraction, reducing the polarization; conversely, large atoms expand the volume and increase the polarization. The determining factor for the polarization change is the gain or loss of space in the polar direction, which results in a change in tetragonality through the coupling to strain.

We find that the contributions from the impurity atom, the charge carriers and the free lattice constants are additive. At 0.125 carriers per u.c., the polarization change ranges from a gain of $25 \%$ to a loss of $100 \%$ (compared to pure $\mathrm{BaTiO}_{3}$ ), depending on the introduced impurity. Which of the contributions dominates depends on the dopant. A general trend can nevertheless be recognized from Fig. 5. For donor dopants, the charge carriers are a dominating factor for the polarization loss, in contrast to acceptor doping, where the chemistry and size of the impurity atom have a prevailing contribution. In both doping regimes, the changes in tetragonality and volume have a strong influence on the system behavior.

Based on our calculations, we can classify the investigated impurity-doped systems with 0.125 carriers per u.c. into one of three categories, based on structural and electronic considerations. In the electron-doped systems, BLTO can be considered to be 
metallic and non-polar. In fact, it has homogeneously spread out electron charge carriers and a centrosymmetric crystal structure, since the polar distortion is completely suppressed. BTNO and BTVO can be regarded as being polar-metal-like with a polaronic conduction mechanism, due to the electron localization on the impurity atom or its axial neighboring Ti along the polar axis. They show both a polar structural distortion, have a non-zero density of states at the Fermi level and the polaronic electron localization could enable polarization switching. The hole-doped systems can also be considered as being polar-metal-like, and in the case of $\mathrm{K}$ doping their polarization is even increased over the undoped case.

\section{Conclusions}

In conclusion, we demonstrated computationally that $\mathrm{BaTiO}_{3}$ can sustain the combination of ferroelectric and metallic properties. With the background-charge method, we found that at low carrier concentrations $(<0.2$ carriers per u.c.), hole doping increases the polarization, whereas electron doping reduces it. At higher concentrations, both electrons and holes reduce the polarization of the system by suppressing the B-site off-centering. The effect of electrons is stronger than that of holes because of the nature of the conduction and valence bands: The electrons strongly affect the Ti ions, whereas the holes spread out over both equatorial and axial oxygens. We found that in impurity-doped systems, multiple structural and electronic factors contribute to the polarization change. The contributions can be separated into effects coming from the chemistry and size of the impurity atom, from the charge carriers and from changes in the shape and volume of the system. These contributions are additive and can overall yield a gain $(\mathrm{K})$ or loss ( $\mathrm{La}, \mathrm{Nb}, \mathrm{V}, \mathrm{Al}, \mathrm{Sc}$ ) of polarization, depending on the introduced dopant. Based on our calculations, we propose a classification of the investigated materials at low doping concentration as either non-polar metallic (La) or polar-metallike ( $\mathrm{Nb}, \mathrm{V}, \mathrm{K}, \mathrm{Al}, \mathrm{Sc})$.

Based on our findings, we propose following experiments to further investigate the interplay between ferroelectricity and metallicity in $\mathrm{BaTiO}_{3}$. Field effect doping could contribute to understanding the effect of charge carriers in $\mathrm{BaTiO}_{3}$, as predicted by our calculations with background charges. Furthermore, doping of $\mathrm{BaTiO}_{3}$ with $\mathrm{Nb}$ or $\mathrm{V}$ could be particularly promising for polarization switchability, which could be allowed by the polaronic localization of the charge carriers in these systems.

\section{Conflicts of interest}

There are no conflicts to declare.

\section{Acknowledgements}

This work was funded by the European Research Council under the European Unions Horizon 2020 research and innovation program Grant Agreement No. 810451, the Körber Foundation and ETH Zürich. V. F. M. was supported by a MARVEL INSPIRE Potentials Master's Fellowship from the NCCR MARVEL, funded by the Swiss National Science Foundation. Calculations were performed on the ETH Zürich Euler cluster. Input and output files can be found at DOI: $10.24435 /$ materialscloud:f4-94.

\section{References}

1 M. E. Lines and A. M. Glass, Principles and Applications of Ferroelectrics and Related Materials, OUP Oxford, Oxford, 2001.

2 P. W. Anderson and E. I. Blount, Phys. Rev. Lett., 1965, 14, 217-219.

3 F. Cordero, F. Trequattrini, F. Craciun, H. T. Langhammer, D. A. B. Quiroga and P. S. Silva Jr., Phys. Rev. B, 2019, 99, 064106.

4 Y. Wang, X. Liu, J. D. Burton, S. S. Jaswal and E. Y. Tsymbal, Phys. Rev. Lett., 2012, 109, 247601.

5 Y. Shi, Y. Guo, X. Wang, A. J. Princep, D. Khalyavin, P. Manuel, Y. Michiue, A. Sato, K. Tsuda, S. Yu, M. Arai, Y. Shirako, M. Akaogi, N. Wang, K. Yamaura and A. T. Boothroyd, Nat. Mater., 2013, 12, 1024-1027.

6 N. A. Benedek and T. Birol, J. Mater. Chem. C, 2016, 4, 4000-4015.

7 T. H. Kim, D. Puggioni, Y. Yuan, L. Xie, H. Zhou, N. Campbell, P. J. Ryan, Y. Choi, J.-W. Kim, J. R. Patzner, S. Ryu, J. P. Podkaminer, J. Irwin, Y. Ma, C. J. Fennie, M. S. Rzchowski, X. Q. Pan, V. Gopalan, J. M. Rondinelli and C. B. Eom, Nature, 2016, 533, 68-72.

8 D. Puggioni, G. Giovannetti, M. Capone and J. M. Rondinelli, Phys. Rev. Lett., 2015, 115, 087202.

9 M. Smidman, M. B. Salamon, H. Q. Yuan and D. F. Agterberg, Rep. Prog. Phys., 2017, 80, 036501.

10 G. H. Kwei, A. C. Lawson, S. J. L. Billinge and S. W. Cheong, J. Phys. Chem., 1993, 97, 2368-2377.

11 T. Kolodiazhnyi, Phys. Rev. B: Condens. Matter Mater. Phys., 2008, 78, 045107.

12 T. Kolodiazhnyi, M. Tachibana, H. Kawaji, J. Hwang and E. Takayama-Muromachi, Phys. Rev. Lett., 2010, 104, 147602.

13 I.-K. Jeong, S. Lee, S.-Y. Jeong, C. J. Won, N. Hur and A. Llobet, Phys. Rev. B: Condens. Matter Mater. Phys., 2011, 84, 064125.

14 E. Iguchi, N. Kubota, T. Nakamori, N. Yamamoto and K. J. Lee, Phys. Rev. B: Condens. Matter Mater. Phys., 1991, 43, 8646-8649.

15 C. Gillot, J. P. Michenaud, M. Maglione and B. Jannot, Solid State Commun., 1992, 84, 1033-1038.

16 K. Page, T. Kolodiazhnyi, T. Proffen, A. K. Cheetham and R. Seshadri, Phys. Rev. Lett., 2008, 101, 205502.

17 Y. Iwazaki, T. Suzuki, Y. Mizuno and S. Tsuneyuki, Phys. Rev. B: Condens. Matter Mater. Phys., 2012, 86, 214103.

18 F. Bruneval, C. Varvenne, J.-P. Crocombette and E. Clouet, Phys. Rev. B: Condens. Matter Mater. Phys., 2015, 91, 024107.

19 D. Hickox-Young, D. Puggioni and J. M. Rondinelli, Phys. Rev. B: Condens. Matter Mater. Phys.,, 2020, 102, 014108. 
20 F. Gu, E. Murray and P. Tangney, Phys. Rev. Mater., 2021, $5,034414$.

21 H. J. Zhao, A. Filippetti, C. Escorihuela-Sayalero, P. Delugas, E. Canadell, L. Bellaiche, V. Fiorentini and J. Íñiguez, Phys. Rev. B, 2018, 97, 054107.

22 G. Kresse and D. Joubert, Phys. Rev. B: Condens. Matter Mater. Phys., 1999, 59, 1758-1775.

23 J. P. Perdew, A. Ruzsinszky, G. I. Csonka, O. A. Vydrov, G. E. Scuseria, L. A. Constantin, X. Zhou and K. Burke, Phys. Rev. Lett., 2008, 100, 136406.

24 R. H. Buttner and E. N. Maslen, Acta Crystallogr., Sect. B: Struct. Sci., 1992, 48, 764-769.

25 R. Dronskowski and P. E. Blöchl, J. Phys. Chem., 1993, 97, 8617-8624.

26 S. Maintz, V. L. Deringer, A. L. Tchougréeff and R. Dronskowski, J. Comput. Chem., 2013, 34, 2557-2567.

27 V. L. Deringer, A. L. Tchougréeff and R. Dronskowski, J. Phys. Chem. A, 2011, 115, 5461-5466.

28 S. Maintz, V. L. Deringer, A. L. Tchougréeff and R. Dronskowski, J. Comput. Chem., 2016, 37, 1030-1035.

29 R. Nelson, C. Ertural, J. George, V. L. Deringer, G. Hautier and R. Dronskowski, J. Comput. Chem., 2020, 41, 1931-1940.

30 S. Maintz, M. Esser and R. Dronskowski, Acta Phys. Pol., B, 2016, 47, 1165.

31 VASP The Guide, https:/cms.mpi.univie.ac.at/vasp/vasp, (accessed October 2020).

32 N. A. Spaldin, J. Solid State Chem., 2012, 195, 2-10.
33 P. Ghosez, X. Gonze, P. Lambin and J.-P. Michenaud, Phys. Rev. B: Condens. Matter Mater. Phys., 1995, 51, 6765-6768.

34 S. F. Yuk, K. C. Pitike, S. M. Nakhmanson, M. Eisenbach, Y. W. Li and V. R. Cooper, Sci. Rep., 2017, 7, 43482.

35 R. Wahl, D. Vogtenhuber and G. Kresse, Phys. Rev. B: Condens. Matter Mater. Phys., 2008, 78, 104116.

36 R. D. King-Smith and D. Vanderbilt, Phys. Rev. B: Condens. Matter Mater. Phys., 1993, 47, 1651-1654.

37 R. Resta, Europhys. Lett., 1993, 22, 133-138.

38 J. M. Rondinelli, A. S. Eidelson and N. A. Spaldin, Phys. Rev. B: Condens. Matter Mater. Phys., 2009, 79, 205119.

39 R. D. Shannon, Acta Crystallogr., Sect. A: Cryst. Phys., Diffr., Theor. Gen. Crystallogr., 1976, 32, 751-767.

40 Database of ionic radii, http://abulafia.mt.ic.ac.uk/shan non/ptable.php, (accessed October 2020).

41 F. D. Morrison, A. M. Coats, D. C. Sinclair and A. R. West, J. Electroceram., 2001, 6, 219-232.

42 A. Janotti, C. Franchini, J. B. Varley, G. Kresse and C. G. V. de Walle, Phys. Status Solidi RRL, 2013, 7, 199-203.

43 H. K. Chandra, K. Gupta, A. K. Nandy and P. Mahadevan, Phys. Rev. B: Condens. Matter Mater. Phys., 2013, 87, 214110.

44 W. Cai, C. Fu, Z. Lin and X. Deng, Ceram. Int., 2011, 37, 3643-3650.

45 C. Collignon, X. Lin, C. W. Rischau, B. Fauqué and K. Behnia, Annu. Rev. Condens. Matter Phys., 2019, 10, 25-44. 